Pure and Applied Mathematics Quarterly

Volume 1, Number 3

(Special Issue: In Memory of

Armand Borel, Part 2 of 3 )

$441-448,2005$

\title{
The Projective Tangent Bundles of a Complex Three-Fold
}

\author{
Friedrich Hirzebruch \\ Dedicated to my friend Armand Borel \\ in memory of the golden fifties in Princeton \\ §1. IntroduCtion AND STATEMENT OF RESUlts.
}

When I visited the Institute for Advanced Study for its 75th anniversary in March 2005, I lectured about my joint work with Armand in the fifties [BH] and reported in particular about an example we studied in $\S 13.9$ and $\S 24.11$. This concerns the 10-dimensional flag manifold

$$
X=\mathbf{U}(4) / \mathbf{U}(2) \times \mathbf{U}(1) \times \mathbf{U}(1) .
$$

The points of $X$ are the ordered triples of one 2-dimensional and two 1dimensional linear subspaces of the standard hermitian space $\mathbb{C}^{4}$ which are pairwise hermitian orthogonal. The manifold $X$ carries two homogenous complex structures, namely the 5 -dimensional complex manifold $X_{1}$ consisting of the flags in $\mathbb{C}^{4}$ of type $(0) \subset(1) \subset(3) \subset(4)$, i. e. the origin contained in a one-dimensional linear subspace, contained in a three-dimensional linear subspace, contained in $\mathbb{C}^{4}$, and the complex manifold $X_{2}$ consisting of flags of type $(0) \subset(1) \subset(2) \subset$ (4). Borel and I prove for the first Chern class $c_{1}$ and the Chern number $c_{1}^{5}$

$$
\begin{aligned}
& c_{1}\left(X_{1}\right) \text { is divisible by } 3 \\
& c_{1}\left(X_{2}\right) \text { is not divisible by } 3
\end{aligned}
$$

$$
\begin{aligned}
& c_{1}^{5}\left[X_{1}\right]=3^{5} \cdot 20=4860 \\
& c_{1}^{5}\left[X_{2}\right]=4500
\end{aligned}
$$

Received September 7, 2005. 
Borel and I did this as an example to our general method to study characteristic classes of homogeneous spaces by using the root systems of Lie groups. As written in $[\mathrm{BH}], \S 24.11, " \ldots$ we get an example of two 5 -dimensional algebraic varieties which are $C^{\infty}$-differentiably homeomorphic, but have different Chern numbers".

E. Calabi attended my Princeton talk and mentioned to me afterwards that $X_{1}$ is the projective covariant tangent bundle of the projective space $\mathbb{P}_{3}(\mathbb{C})$, and $X_{2}$ is the projective contravariant tangent bundle of $\mathbb{P}_{3}(\mathbb{C})$. Indeed, the points of the projective covariant bundle consist of a point $x$ in $\mathbb{P}_{3}(\mathbb{C})$ and a plane going through $x$ which as a tangent plane in $x$ is annihilated by a covariant tangent vector in $x$. This corresponds to the inclusion $(1) \subset$ (3). Similarly $(1) \subset(2)$ describes the projective contravariant tangent bundle.

Motivated by the remark of Calabi I studied the following problem:

Let $B$ be a compact complex manifold of dimension 3 and $c=1+c_{1}+c_{2}+c_{3}$ its total Chern class $\left(c_{i} \in H^{2 i}(B, \mathbb{Z})\right)$. Let $X_{1}, X_{2}$ be the projective covariant tangent bundle of $B$ and the projective contravariant tangent bundle respectively. Then $X_{1}, X_{2}$ are 5-dimensional compact complex manifolds fibred over $B$ with the projective plane as fibre. After introducing a hermitian metric in $B$ the covariant and contravariant tangent bundles with structural group $\mathbf{U}(3)$ are anti-isomorphic by complex conjugation in each fibre. Therefore $X_{1}$ and $X_{2}$ are diffeomorphic. The cohomology ring $H^{*}(B, \mathbb{Z})$ maps isomorphically into the cohomology ring of $X_{1}$ and $X_{2}$ respectively and $H^{*}\left(X_{i}, \mathbb{Z}\right)$ is an extension of $H^{*}(B, \mathbb{Z})$ by the first Chern class $\eta$ of the tautological line bundle over $X_{i}$. The total Chern classes of $X_{1}, X_{2}$ will be denoted by

$$
d=1+d_{1}+d_{2}+d_{3}+d_{4}+d_{5} \text { where } d_{j} \in H^{2}\left(X_{i}, \mathbb{Z}\right)
$$

Each Chern number of $X_{1}, X_{2}$ can be calculated as a linear combination of the Chern numbers $c_{1}^{3}, c_{1} c_{2}, c_{3}$ of $B$. I did not carry this out completely. I formulate here only the following result for $d_{1}$ and the Chern number $d_{1}^{5}$.

Proposition 1. We have

$$
\begin{aligned}
& d_{1}\left(X_{1}\right)=-3 \eta \\
& d_{1}\left(X_{2}\right)=2 c_{1}-3 \eta \\
d_{1}^{5}\left[X_{1}\right]= & 3^{5}\left(c_{1}^{3}-2 c_{1} c_{2}+c_{3}\right)[B] \\
d_{1}^{5}\left[X_{2}\right]= & 9\left(23 c_{1}^{3}-36 c_{1} c_{2}-27 c_{3}\right)[B]
\end{aligned}
$$

Of course, (1) and (2) follow from (3) and (4). Let $g \in H^{2}\left(\mathbb{P}_{3}(\mathbb{C}), \mathbb{Z}\right)$ be the positive generator of the cohomology ring. The total Chern class of $\mathbb{P}_{3}(\mathbb{C})$ is 
$(1+g)^{4}$, and the Chern numbers are

$$
c_{1}^{3}=64, c_{1} c_{2}=24, c_{3}=4,
$$

and (4) gives the values 4860 and 4500 respectively.

We now specialize to the case that the complex 3 -fold $B$ is a Calabi-Yau manifold, i. e. we assume that the first Chern class of $B$ vanishes. Then we only have the Chern number $c_{3}[B]$ which is the Euler number of $B$ and all Chern numbers of $X_{1}$ and $X_{2}$ are multiples of $c_{3}[B]$. We have the following table for $X_{1}, X_{2}$ and the Cartesian product $B \times \mathbb{P}_{2}(\mathbb{C})$.

\begin{tabular}{c|c|c|c} 
& $X_{1}$ & $X_{2}$ & $B \times \mathbb{P}_{2}(\mathbb{C})$ \\
\hline$d_{5}$ & $3 c_{3}$ & $3 c_{3}$ & $3 c_{3}$ \\
$d_{4} d_{1}$ & $9 c_{3}$ & $9 c_{3}$ & $9 c_{3}$ \\
$d_{3} d_{2}$ & $3 c_{3}$ & $3 c_{3}$ & $3 c_{3}$ \\
$d_{3} d_{1}^{2}$ & $9 c_{3}$ & $9 c_{3}$ & $9 c_{3}$ \\
$d_{2}^{2} d_{1}$ & $27 c_{3}$ & $-27 c_{3}$ & 0 \\
$d_{2} d_{1}^{3}$ & $81 c_{3}$ & $-81 c_{3}$ & 0 \\
$d_{1}^{5}$ & $243 c_{3}$ & $-243 c_{3}$ & 0
\end{tabular}

If the Euler number $c_{3}[B]$ is not zero, then $X_{1}, X_{2}$ have different Chern numbers, therefore they are not biholomorphically equivalent. The complex cobordism class of a compact complex manifold is determined by its Chern numbers $[\mathrm{M}]$. Therefore the complex cobordism classes of $X_{1}$ and $X_{2}$ are equal if and only if $c_{3}[B]=0$. From table (5) we obtain

Proposition 2. For the projective covariant or contravariant tangent bundles $X_{1}, X_{2}$ of the Calabi-Yau manifold $B$ we have in the complex cobordism ring the equation

$$
X_{1}+X_{2}=2 B \times \mathbb{P}_{2}(\mathbb{C})
$$

Remarks:

(1) The results (5) and (6) are true if we only assume that the Chern numbers $c_{1}^{3}$ and $c_{1} c_{2}$ of $B$ vanish. 
(2) The Todd genus is multiplicative in projective bundles ([H1] and [H3]). The Todd genus of $B$ equals $\frac{1}{24} c_{1} c_{2}[B]=0$. Hence the Todd genus of $X_{1}$ and $X_{2}$ vanishes. For a 5 -fold $Y$ with Chern classes $d_{i}$ the Todd genus is given by $([\mathrm{H} 3], \S 1)$

$$
T(Y)=\frac{1}{1440}\left(-d_{4} d_{1}+d_{3} d_{1}^{2}+3 d_{2}^{2} d_{1}-d_{2} d_{1}^{3}\right)[Y]
$$

which indeed vanishes for $X_{1}, X_{2}$ and $B \times \mathbb{P}_{2}(\mathbb{C})$, see table (5).

\section{$\S 2$. Proofs}

Let $E$ be a complex vector bundle (fibre $\mathbb{C}^{n}$, structural group $\mathbf{U}(n)$ ) over the compact manifold $B$ with total Chern class

$$
1+a_{1}+\ldots+a_{n} \quad, \quad a_{i} \in H^{2 i}(B, \mathbb{Z}) .
$$

Consider the associated projective bundle $X$ over $B$ with fibre $\mathbb{P}_{n-1}(\mathbb{C})$ and projection $\pi: X \rightarrow B$. We have

$$
\pi^{*} E=L \oplus \bar{E}
$$

where $L$ is the tautological line bundle over $X$ and $\bar{E}$ (fibre $\mathbb{C}^{n-1}$ ) its hermitian orthogonal complement. Then $\bar{E} \otimes L^{-1}$ is the tangential vector bundle $T$ along the fibres of $X$ (see [H3], §13) and

$$
\pi^{*} E \otimes L^{-1}=T \oplus 1
$$

where 1 denotes the trivial line bundle. We always use tacitly that $\pi^{*}$ maps the integral cohomology ring of $B$ isomorphically into $H^{*}(X, \mathbb{Z})$ and usually omit $\pi^{*}$ in the notation. The ring $H^{*}(X, \mathbb{Z})$ is an extension of $H^{*}(B, \mathbb{Z})$ by the first Chern class $\eta \in H^{2}(X, \mathbb{Z})$ of the tautological line bundle $L$ over $X$. The total Chern class of $\pi^{*} E$ can be split formally (or by lifting $E$ to the associated bundle with $\mathbf{U}(n) / \mathbf{U}(1)^{n}$ as fibre)

$$
1+a_{1}+\ldots+a_{n}=\left(1+x_{1}\right) \ldots\left(1+x_{n}\right) \quad, \quad x_{i} \in H^{2}
$$

and (1) implies that the total Chern class of $T$ equals

$$
\left(1+x_{1}-\eta\right) \ldots\left(1+x_{n}-\eta\right)=\sum_{i=0}^{n}(1-\eta)^{n-i} a_{i}
$$

and, since the $n$-th Chern class of $T$ is zero, 


$$
\sum_{i=0}^{n}(-\eta)^{n-i} a_{i}=0 \quad \text { (formula of Guy Hirsch) }
$$

The ring $H^{*}(X, \mathbb{Z})$ is the extension of $H^{*}(B, \mathbb{Z})$ by $\eta$ with the relation (3). Thus $H^{*}(X, \mathbb{Z})$ is a free module over $H^{*}(B, \mathbb{Z})$ with base $1, \eta, \ldots, \eta^{n-1}$.

All this is already mentioned in [H1] and in the following paper [H2] where the total Chern class of the flag manifold $\mathbf{U}(n) / \mathbf{U}(1)^{n}$ is calculated. This is the beginning of the joint work with A. Borel. When I told him in 1953 about the formula for $\mathbf{U}(n) / \mathbf{U}(1)^{n}$ he pointed out that this can be generalized to $G / T$ where $G$ is a compact connected Lie group and $T$ a maximal torus of $G$ (see [H2], remark at the end of $\S 7$ ).

If $B$ is a complex manifold of dimension $m$ with total Chern class

$$
c(B)=1+c_{1}+c_{2}+\ldots c_{m}
$$

then

$$
c(X)=c(B) \cdot \sum_{i=0}^{n}(1-\eta)^{n-i} a_{i}
$$

To calculate the Chern numbers of $X$ we use that $-\eta$ when restricted to the fibre $\mathbb{P}_{n-1}(\mathbb{C})$ over $X$ is the positive generator of $H^{2}\left(\mathbb{P}_{n-1}(\mathbb{C}), \mathbb{Z}\right) \simeq \mathbb{Z}$ represented by a hyperplane.

We denote the total Chern class $c(X)$ by $d$

$$
d=1+d_{1}+\ldots+d_{m+n-1}
$$

A Chern number $d_{r_{1}} d_{r_{2}} \ldots d_{r_{s}}\left(r_{1}+r_{2}+\ldots+r_{s}=m+n-1\right)$ equals by (3) and (4) a linear combination of monomials of dimension $m$ in the $a_{i}$ and $c_{j}$ multiplied with $(-\eta)^{n-1}$. Since $(-\eta)^{n-1}$ gives 1 when evaluated on the fibre, the Chern number $d_{r_{1}} d_{r_{2}} \ldots d_{r_{s}}[X]$ equals the above polynomial in $a_{i}$ and $c_{j}$ of dimension $m$ evaluated on $B$.

We now carry this out in the cases we are interested in.

We specialize to $m=n=3$. The Hirsch relation (3) gives

$$
\eta^{3}=\eta^{2} a_{1}-\eta a_{2}+a_{3}
$$


and by repeated applications of (5)

$$
\begin{gathered}
\eta^{4}=\eta^{2}\left(a_{1}^{2}-a_{2}\right)+\eta\left(-a_{1} a_{2}+a_{3}\right) \\
\eta^{5}=\eta^{2}\left(a_{1}^{3}-2 a_{1} a_{2}+a_{3}\right)
\end{gathered}
$$

We now consider the covariant tangent bundle of the 3-dimensional complex manifold $B$ with Chern classes $c_{1}, c_{2}, c_{3}$. The Chern classes of the covariant tangent bundle of $B$ are $-c_{1}, c_{2},-c_{3}$. According to (4), the total Chern class of the projective covariant tangent bundle $X_{1}$ equals

$$
\begin{aligned}
d & =\left(1+c_{1}+c_{2}+c_{3}\right)\left((1-\eta)^{3}-(1-\eta)^{2} c_{1}+(1-\eta) c_{2}-c_{3}\right) \\
d_{1} & =-3 \eta
\end{aligned}
$$

We have by (5")

$$
d_{1}^{5}=-3^{5} \eta^{2}\left(-c_{1}^{3}+2 c_{1} c_{2}-c_{3}\right)
$$

Thus the first formula in (4) of $\S 1$ is proved.

If we take the contravariant tangent bundle of $B$, then by definition its Chern classes are those of $B$. The total Chern class $d$ of the associated projective bundle $X_{2}$ is now given by

$$
\begin{aligned}
d & =\left(1+c_{1}+c_{2}+c_{3}\right)\left((1-\eta)^{3}+(1-\eta)^{2} c_{1}+(1-\eta) c_{2}+c_{3}\right) \\
d_{1} & =2 c_{1}-3 \eta
\end{aligned}
$$

For the calculation of $d_{1}^{5}=\left(2 c_{1}-3 \eta\right)^{5}$ we have to replace in this expression $\eta^{3}, \eta^{4}, \eta^{5}$ by the quadratic polynomials in $\eta$ given in (5), (5'), (5") with $a_{i}=c_{i}$.

We have

$$
\left(2 c_{1}-3 \eta\right)^{5}=9\left(-27 \eta^{5}+90 c_{1} \eta^{4}-120 c_{1}^{2} \eta^{3}+80 c_{1}^{3} \eta^{2}\right)
$$


and by $(5),\left(5^{\prime}\right),\left(5^{\prime \prime}\right)$

$$
\begin{aligned}
\eta^{5} & =\left(c_{1}^{3}-2 c_{1} c_{2}+c_{3}\right) \eta^{2} \\
c_{1} \eta^{4} & =\left(c_{1}^{3}-c_{1} c_{2}\right) \eta^{2} \\
c_{1}^{2} \eta^{3} & =c_{1}^{3} \eta^{2}
\end{aligned}
$$

which gives the second formula in (4) of $\S 1$.

We now assume that $B$ is a Calabi-Yau manifold $\left(c_{1}=0\right)$. The Chern class $c_{2}$ cannot occur in the final result because $c_{1} c_{2}=0$. Therefore, for the calculation we put $c_{2}=0$ and have in the covariant case $\eta^{3}=-c_{3}$ and for the total Chern class of $X_{1}$

$$
\begin{gathered}
d\left(X_{1}\right)=\left(1+c_{3}\right)\left(1-3 \eta+3 \eta^{2}\right) \\
d_{1}=-3 \eta, d_{2}=3 \eta^{2}, d_{3}=c_{3}, d_{4}=-3 c_{3} \eta, d_{5}=3 c_{3} \eta^{2}
\end{gathered}
$$

The values in table (5) of $\S 1$ are now easily obtained.

For the projective contravariant bundle $X_{2}$ we have (again mod $c_{2}$ ) the same formulas as in (9) and (10), but in this case

$$
\eta^{3}=c_{3}
$$

This checks with table 5 in $\S 1$. For the Cartesian product $B \times \mathbb{P}_{2}(\mathbb{C})$ again $(9)$ and (10) hold, but $\eta^{3}=0$ and the values in table 5 result.

\section{$\underline{\text { Remark: }}$}

Proposition 2 and table (5) of $\S 1$ can be generalized to higher dimensions. We assume that $B$ is a compact almost complex manifold of dimension $n$ and that all Chern numbers of $B$ vanish except $c_{n}[B]$, the Euler number. Consider the projective covariant tangent bundle $X_{1}$ of $B$ and the projective contravariant tangent bundle $X_{2}$ of $B$. Both $X_{1}$ and $X_{2}$ are fibred over $B$ with $\mathbb{P}_{n-1}(\mathbb{C})$ as fibre. The Chern classes of $X_{1}, X_{2}$ and $B \times \mathbb{P}_{n-1}(\mathbb{C})$ are denoted $d_{i}$. Using the preceding methods, the following is proved easily.

If $n$ is even, then $X_{1}$ and $X_{2}$ have equal Chern numbers, hence $X_{1}=X_{2}$ in the complex cobordism ring. 
If $n$ is odd, the following holds:

If a Chern number is a monomial of dimension $2 n-1$ in Chern classes $d_{i}$ with all $i<n$, then for $X_{2}$ it is minus the corresponding Chern number of $X_{1}$, whereas it vanishes for $B \times \mathbb{P}_{n-1}(\mathbb{C})$. The other Chern numbers agree on $X_{1}, X_{2}$, and $B \times \mathbb{P}_{n-1}(\mathbb{C})$. Hence we have in the complex cobordism ring

$$
X_{1}+X_{2}=2 B \times \mathbb{P}_{n-1}(\mathbb{C}) .
$$

\section{REFERENCES}

[BH] A. Borel and F. Hirzebruch, Characteristic classes and homogeneous spaces, Amer. J. Math. 80(1958), 458-538(part1), 81(1959), 315-382(part II), 82(1960), 491-504(part III). Also contained in A. Borel, Oeuvres, Springer 1983, and in F. Hirzebruch, Gesammelte Abhandlungen, Springer 1987

[H1] F. Hirzebruch, Todd arithmetic genus for almost complex manifolds, mimeographed notes, Princeton University 1953, published as Nr. 4 in Vol. 1 of the Gesammelte Abhandlungen

[H2] F. Hirzebruch, The index of an oriented manifold and the Todd genus of an almost complex manifold, mimeographed notes, Princeton University 1953, published as Nr. 6 in Vol. 1 of the Gesammelte Abhandlungen

[H3] F. Hirzebruch, Neue topologische Methoden in der algebraischen Geometrie, Springer 1956

[M] J. W. Milnor, On the cobordism ring $\Omega^{*}$ and a complex analogue, Part I. Amer. J. Math. 82(1960), 505-521

Friedrich Hirzebruch

MPI für Mathematik, Vivatsgasse 7, D-53111, Bonn, Germany.

E-Mail: hirzebruch@mpim-bonn.mpg.de 